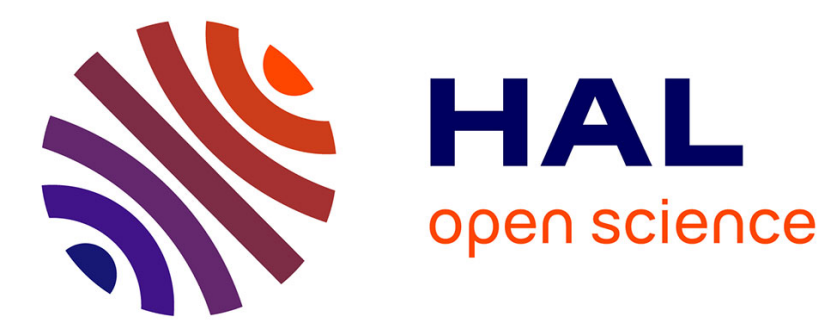

\title{
Dual-color fluorescence lifetime correlation spectroscopy to quantify protein-protein interactions in live cell.
} Sergi Padilla-Parra, Nicolas Audugé, Maïté Coppey-Moisan, Marc Tramier

\section{To cite this version:}

Sergi Padilla-Parra, Nicolas Audugé, Maïté Coppey-Moisan, Marc Tramier. Dual-color fluorescence lifetime correlation spectroscopy to quantify protein-protein interactions in live cell.. Microscopy Research and Technique, 2011, 74 (8), pp.788-93. 10.1002/jemt.21015 . inserm-00604664

\section{HAL Id: inserm-00604664 https://www.hal.inserm.fr/inserm-00604664}

Submitted on 30 Jun 2011

HAL is a multi-disciplinary open access archive for the deposit and dissemination of scientific research documents, whether they are published or not. The documents may come from teaching and research institutions in France or abroad, or from public or private research centers.
L'archive ouverte pluridisciplinaire HAL, est destinée au dépôt et à la diffusion de documents scientifiques de niveau recherche, publiés ou non, émanant des établissements d'enseignement et de recherche français ou étrangers, des laboratoires publics ou privés. 


\section{Dual color Fluorescence Lifetime Correlation Spectroscopy to quantify protein-protein interactions in live cell}

Sergi Padilla-Parra, Nicolas Audugé, Maïté Coppey-Moisan and Marc Tramier

Institut Jacques Monod, UMR 7592, CNRS, Université Paris-Diderot, Bâtiment Buffon, 15 rue Hélène Brion, 75013 Paris, France

Correspondence: $\quad$ Marc Tramier marc.tramier@univ-rennes1.fr, tel: +33223235487

Maité Coppey-Moisan coppey.maite@ijm.univ-paris-diderot.fr, tel: +33157278041

SP-P present address: Emory University, Department of Pediatrics, Infectious Diseases, 2015 Uppergate Dr., Atlanta, GA 30322

MT present address: Institut de Génétique et Développement de Rennes, UMR 6061, CNRS, Université de Rennes 1, 2 Av. du Pr Léon Bernard, 35043 Rennes Cedex, France

Keywords: FCS, FCCS, FLCS, quantitative fluorescence microscopy, TCSPC

Running title: Dual color FLCS 


\begin{abstract}
Dual color FCS is an interesting method to quantify protein interaction in living cells. But, when performing these experiments, one must compensate for a known spectral bleed through artifact that corrupts cross-correlation data. In this article, problems with crosstalk were overcome with an approach based on Fluorescence Lifetime Correlation Spectroscopy (FLCS). We show that FLCS applied to dual color EGFP and mCherry cross-correlation allows the determination of protein-protein interactions in living cells without the need of spectral bleed through calibration. The methodology was validated by using EGFP-mCherry tandem in comparison to co-expressed EGFP and mCherry in live cell. The dual color FLCS experimental procedure where the different laser intensities do not have to be controlled during experiment is really very helpful to study quantitatively protein interactions in live sample.
\end{abstract}




\section{INTRODUCTION}

Fluorescence microscopy techniques are commonly used to monitor protein-protein interactions in living cells taking advantage of the detection of Förster Resonance Energy Transfer (FRET) (Förster, 1948). Techniques based on the calculation of the lifetime such as Fluorescence Lifetime Imaging Microscopy (FLIM) (Wouters and Bastiaens, 1999; Tramier et al., 2002; Padilla-Parra et al., 2008) or intensity based ratiometric approaches (Gordon et al., 1998; Berney and Danuser, 2003; Elangovan et al., 2003; Stockholm et al., 2004) have been widely used to detect FRET by the biological community over the last decade. The main limitation, however, to use FRET is the requirement of the close proximity of the two fluorescent tags. Dual color Fluorescence Correlation Spectroscopy (FCS) is an interesting alternative to deal with this limitation (Bacia et al., 2002). The main idea is to follow the codiffusion of two proteins labeled with differently colored fluorescent tags by analyzing the cross-correlation of temporal fluorescence intensity fluctuations. The most common couples of protein tags used (ECFP/EYFP and EGFP/mCherry), however, present sufficient overlap in their emission spectra to give rise to spectral bleed through, an artifact that corrupts the crosscorrelation data (Bacia and Schwille, 2007). The contribution of the cross-talk from the shorter wavelengths emission species in the longer wavelengths channel is usually minimized by decreasing the intensity of the first laser. The cross-talk can also be calibrated and its amount retrieves during data analysis (Bacia and Schwille, 2007). Recently, this approach was used in live cells and brought novel informations on a number of macromolecular complexes, as the MAP kinase complexes in yeast pheromone signaling (Maeder et al., 2007), the dimerization of EGF receptors (Liu et al., 2007), the T cell receptor complexes (Lillemeier et al., 2010), the estrogen receptor interactions and dynamics (Savatier et al., 2010), and the virus RNA polymerase assembly (Huet et al., 2010). But nonetheless at least $10 \%$ of crosstalk is always observed when working with green and red fluorescent proteins, that impedes the study of very low amounts of protein-protein interactions. The development of novel fluorescent proteins (Kogure et al., 2006), decreases the contribution of the spectral cross-talk to the cross-correlation, but the weak photo stability of some of them introduces other difficulties.

A way of getting rid of the bleed-through is the so-called switching method or pulsed interleaved excitation (Müller et al., 2005; Thews et al., 2005; Takahashi et al., 2008). In this method, two alternating laser beams are controlled by an acousto-optic modulator and the synchronized correlation signals in both detectors are calculated as cross-correlation functions with no cross-talk. A similar method was recently implemented in dual color scanning FCS with a conventional confocal microscope to quantify ligand-receptor interactions in cell membranes of living zebrafish embryos (Ries et al., 2009).

Here, we overcome the spectral cross-talk with an approach based on Fluorescence Lifetime Correlation Spectroscopy (FLCS). The combination of TCSPC and FCS (FLCS) (Bohmer et al., 2002; Kapusta et al., 2007) takes advantage of time resolved photon detection by using a pulsed laser to differentiate the contribution of two different species to the FCS trace. Each fluorescent component coming from the sample can be resolved on the basis of its fluorescence decay signature. FLCS was already used in vitro to suppress scattered light and detector after-pulses (Kapusta et al., 2007), to study dynamic transition between two fluorescence lifetime states (Gregor and Enderlein, 2007), to resolve different lifetime species (Ray et al., 2008) and to measure precise concentration values as low as picomolar of fluorophore (Rüttinger et al., 2010). Recently, FLCS was employed in live cells to quantify the interaction of EGFR neutralizing antibody labeled with Alexa488 and EGFR-GFP by 
fluorescence lifetime cross-correlation measurement (Chen and Irudayaraj, 2010). Here, we show that FLCS applied to dual color EGFP and mCherry cross-correlation allows the determination of protein-protein interactions in living cells without the need of the spectral bleed through calibration. The advantage of the methodology was established as being independent of the respective amount of fluorescence of both species by performing experiments with EGFP-mCherry tandem and co-expressed free GFP and mCherry at different concentrations in live cell. 


\section{MATERIAL AND METHODS}

\section{Plasmid constructs and cell culture}

The plasmid coding the mCherry-EGFP tandem has been described elsewhere (Tramier et al., 2006). He-La cells were cultured in Dulbecco's Modified Eagle Medium containing 10\% fetal bovine serum (PAA Laboratories $\mathrm{GmbH}$, Pasching, Austria). The cultures were incubated at $37{ }^{\circ} \mathrm{C}$ in a humidified atmosphere of $5 \% \mathrm{CO}_{2}$. He-La cells were seeded on $32 \mathrm{~mm}$ round glass coverslip at a density of $2 \times 10^{5}$ cells. When cells were about $50-70 \%$ of confluence, they were transfected by EGFP and mCherry expressing plasmids or by mCherry-EGFP tandem expressing plasmid with a total amount of $1 \mu \mathrm{g}$ of expression vectors using Nanofectin I (PAA). 24 hours after transfection, coverslips were mounted in an open observation chamber with special DMEM-F12 to prevent fluorescence from the medium (DMEM-F12 without Phenol red, B12 vitamin, Riboflavin and supplemented with $20 \mathrm{mM}$ Hepes and L-Glutamine from PAA). A microscope incubation chamber (PeCon $\mathrm{GmbH})$ was used to perform live cell measurements at $37^{\circ} \mathrm{C}$.

\section{Fluorescence Lifetime Correlation Spectroscopy microscopy}

The configuration of the scanning confocal time-resolved microscope MicroTime 200 (Picoquant, Berlin, Germany) used in this work is presented Figure 1A. Briefly, two laser excitation sources (a pulsed diode laser operating at $470 \mathrm{~nm}$ (Picoquant) and a continuous DPSS laser operating at $561 \mathrm{~nm}$ (Coherent)) are focused at the center of the optical axis in the sample through a water objective with a high numerical aperture objective $(\times 60$, NA 1.3$)$ mounted on an inverted microscope (Olympus, Tokyo, Japan). The fluorescence emission passes through a double dichroic mirror (DM 470/571 nm) and is focused on a pinhole, and a second dichroic mirror (DM $550 \mathrm{~nm})$ splits the radiation into two channels. Two avalanche photodiodes (SPADs 14, Perkin Elmer) in front of which are placed the emission filters 525 +/- $25 \mathrm{~nm}$ and $593+/-20 \mathrm{~nm}$, constitute the green and red detectors, respectively. The subcellular position of the sampled volume is chosen by first acquiring a pre-scan image of the field of view. A piezo displacement of the objective in the range of $80 \times 80 \mu \mathrm{m}$ can be carried out (P-733 Piezo-positioner, PI). The TimeHarp 300 PC board (PicoQuant) is employed in a time-tagged time-resolved (TTTR) mode and is coupled to a PC that pilots the data acquisition and analysis using the Symphotime software (PicoQuant). Single photon counting Time-Tagged Time-Resolved (TTTR) trace was acquired during 60 seconds. After lifetime filter calculation, auto- and cross-correlation functions of both green and red channels are computed using the Symphotime software following Eq. 3, 4 and 5. Fit were carried out with a single species Brownian diffusion model to recover amplitudes ( $\mathrm{Gg}$, $\mathrm{Gr}$ and $\mathrm{Ggr}$ ) and apparent diffusion coefficients $\left(D_{g}, D_{r}\right.$ and $\left.D_{g r}\right)$ The beam waist, $\omega_{0}$, was determined using the point spread function of the confocal system ( $260 \mathrm{~nm}$ in the green channel and $320 \mathrm{~nm}$ in the red channel). For the fit, $\omega_{0}$ is fixed to $260 \mathrm{~nm}$ (the smallest volume) and the structural parameter, $\mathrm{z} / \omega_{0}$ to 4 (corresponding to a volume of $0.4 \mathrm{fl}$ ). 


\section{RESULTS AND DISCUSSION}

\section{Dual color FLCS methodology}

The use of a pulsed laser to perform FCS takes advantage of Time Correlated Single Photon Counting (TCSPC) to differentiate the contribution of two different fluorescence lifetime species to FCS file (FLCS, Bohmer et al., 2002). Indeed, each single photon is recorded with its global arrival time and the delay time with respect to the corresponding laser pulse. While the delay time is evaluated in fluorescence lifetime related analysis, the global arrival time can be used to form a fluorescence intensity time trace (Time-Tagged Time-Resolved (TTTR) file), making all related analysis possible. Sorting the photon events of a TTTR file into subsequent time bins, allows FCS calculation, time trace TCSPC fitting, time-gated FCS. The FLCS method relies on the calculation of lifetime filters for each species having different fluorescence decay signature in TCSPC histogram. For each time-resolved channel, the lifetime filter value gives the statistical weight of the counted photon for its corresponding species. These different filters can be selected during auto- and cross-correlation process of the absolute time traces to take into account the different weighting of each individual photon.

By using two laser to excite simultaneously two spectrally different fluorophores, a pulsed laser (470 nm which can excite a green fluorescent species, i.e. EGFP) and a continuous laser (561 to excite a red fluorescent protein,i.e. mCherry), dual color FLCS provides a way to remove the spectral bleed through of the green species into the red detection channel (configuration of the system is presented in Fig.1-A). The "green" photons detected in the red channel are distinguished from the "red" ones because they are time correlated to the pulsed laser (see the fluorescence decay histogram in Fig.1-B top) in contrast to the "red" photons which do not harbor any fluorescence decay signature (see the constant emission histogram in Fig.1-B, middle). When both fluorophores are excited together, the fluorescence decay into the red channel (Fig.1-B bottom) corresponds to the spectral bleed through of the green species, although the non-correlated trace (constant histogram) is the signature of the red emission. In practice, "lifetime filter" functions, $f_{a}^{r}(i)$ and $f_{b}^{r}(i)$, where $i$ correspond to the time-resolved channel (in ns), are calculated from the two components fluorescence decay analysis acquired in the red channel as described below. Shortly, the fluorescence decay histogram, Decay ${ }^{\mathrm{r}}$ (i) (Fig.2-A, black curve), corresponding to the red channel detection of simultaneous pulsed $470 \mathrm{~nm}$ and CW $560 \mathrm{~nm}$ excitation of EGFP and mCherry fluorophores can be expressed as a linear combination of two normalized histograms:

$$
\operatorname{Decay}^{\mathrm{r}}(\mathrm{i})=\mathrm{w}_{\mathrm{a}}^{\mathrm{r}} \cdot \mathrm{a}(\mathrm{i})+\mathrm{w}_{\mathrm{b}}^{\mathrm{r}} \cdot \mathrm{b}(\mathrm{i})
$$

With a (i) $=\mathrm{A}(\mathrm{i}) / \Sigma \mathrm{A}(\mathrm{i})$ and $\mathrm{b}(\mathrm{i})=\mathrm{B}(\mathrm{i}) / \Sigma \mathrm{B}(\mathrm{i}), \mathrm{A}(\mathrm{i})$ and $\mathrm{B}(\mathrm{i})$ corresponding to the histograms of the EGFP fluorescence decay species (Fig.2-A, blue curve) and of the steady-state fluorescence of mCherry (Fig.2-A, constant histogram in pink), respectively. $\mathrm{w}_{\mathrm{a}}^{\mathrm{r}}$ and $\mathrm{w}_{\mathrm{b}}^{\mathrm{r}}$ are the amplitudes of the photon count contribution of blue and pink histogram species. These parameters are recovered from the adjustment of the right part of Eq. 1 to the experimental decay (residue of the adjustment displayed in light blue in Fig 2 A).

The corresponding lifetime filters functions, $\mathrm{f}_{\mathrm{a}}^{\mathrm{r}}(\mathrm{i})$ and $\mathrm{f}_{\mathrm{b}}^{\mathrm{r}}(\mathrm{i})$ are defined as follow:

$$
\left\langle\Sigma \mathrm{f}_{\mathrm{a}}^{\mathrm{r}}(\mathrm{i}) \cdot \operatorname{Decay}^{\mathrm{r}}(\mathrm{i})\right\rangle=\mathrm{w}_{\mathrm{a}}^{\mathrm{r}} \text { and }\left\langle\sum \mathrm{f}_{\mathrm{b}}^{\mathrm{r}}(\mathrm{i}) \cdot \operatorname{Decay}^{\mathrm{r}}(\mathrm{i})\right\rangle=\mathrm{w}_{\mathrm{b}}^{\mathrm{r}}
$$


where the brackets denote averaging over infinite number of measurements. The numerical calculation of these filters is described in details elsewhere (Böhmer et al., 2002). In Fig.2-B are shown the filter corresponding to the fluorescence decay species (in blue, $\mathrm{f}_{\mathrm{a}}^{\mathrm{r}}(\mathrm{i})$ ) and this corresponding to the steady-state fluorescence (in pink, $\mathrm{f}_{\mathrm{b}}^{\mathrm{r}}(\mathrm{i})$ ),. Note that, for photons tagged with a time-resolved value comprised between 2 and $10 \mathrm{~ns}$ (corresponding to the fluorescence decay duration) the respective statistical weight is larger for the fluorescence decay species, whereas it is larger for the steady-state fluorescence species for photons tagged with other time value.

The auto $(\operatorname{Gg}(\tau), \operatorname{Gr}(\tau))$ and cross-correlation $(\operatorname{Ggr}(\tau))$ functions are directly calculated by taking into account the contribution of each species with its filter, in both green and red channels:

$$
\begin{aligned}
& \operatorname{Gg}(\tau)=\left\langle\left(\sum \mathrm{f}_{\mathrm{a}}^{\mathrm{g}}(\mathrm{i}) \cdot \mathrm{I}_{\mathrm{i}}^{\mathrm{g}}(\mathrm{t})\right)\left(\sum \mathrm{f}_{\mathrm{a}}^{\mathrm{g}}(\mathrm{i}) \cdot \mathrm{I}_{\mathrm{i}}^{\mathrm{g}}(\mathrm{t}+\tau)\right)\right\rangle \\
& \operatorname{Gr}(\tau)=\left\langle\left(\sum \mathrm{f}_{\mathrm{b}}^{\mathrm{r}}(\mathrm{i}) \cdot \mathrm{I}_{\mathrm{i}}^{\mathrm{r}}(\mathrm{t})\right)\left(\sum \mathrm{f}_{\mathrm{b}}^{\mathrm{r}}(\mathrm{i}) \cdot \mathrm{I}_{\mathrm{i}}^{\mathrm{r}}(\mathrm{t}+\tau)\right)\right\rangle \\
& \operatorname{Ggr}(\tau)=\left\langle\left(\sum \mathrm{f}_{\mathrm{a}}^{\mathrm{g}}(\mathrm{i}) \cdot \mathrm{I}_{\mathrm{i}}^{\mathrm{g}}(\mathrm{t})\right)\left(\sum \mathrm{f}_{\mathrm{b}}^{\mathrm{r}}(\mathrm{i}) \cdot \mathrm{I}_{\mathrm{i}}^{\mathrm{r}}(\mathrm{t}+\tau)\right)\right\rangle
\end{aligned}
$$

where $\mathrm{I}_{\mathrm{i}}^{\mathrm{g}}$ and $\mathrm{I}_{\mathrm{i}}^{\mathrm{r}}(\mathrm{t})$ are the fluorescence signal in the ith time-resolved channel at measurement time $t$ in the green and red detection channels, respectively and $f_{a}{ }^{g}(i)$ is the lifetime filter of the fluorescence decay of EGFP in the green channel, $\mathrm{f}_{\mathrm{b}}{ }^{\mathrm{r}}(\mathrm{i})$ being the filter of the steady-state fluorescence of mCherry in the red channel.

\section{FLCS remove EGFP spectral bleed through into mCherry detection channel}

The efficient removal of spectral bleed through from EGFP emission into the red detection channel by dual-color FLCS was validated on co-transfected cells expressing free EGFP and mCherry. The cross-correlation curves calculated without (black) and with the lifetime filters (blue), displayed in Fig.3-A, show the efficiency of the use of FLCS to get rid of the green fluorescence detected into the red channel. In the present example, for a similar amount of green and red fluorescence, as revealed from the auto-correlation amplitudes of both species (green and red curves), the contribution of the cross-talk is about $25 \%$. This value is similar to the amplitude of the cross-correlation measured when EGFP and mCherry are linked together in the tandem molecule (Fig.3-B, black curve: without FLCS). However, applying the lifetime filters to the calculation of the cross-correlation completely abolishes the cross-correlation for free EGFP and mCherry and leads to a significant amount of cross-correlation amplitude for the tandem (Fig.3-B, blue curve). Importantly, the correction of the spectral bleed through by FLCS is independent of the respective green and red fluorescence intensities, and thus to the percentage of spectral bleed through in the red detector channel. Indeed, we show that decreasing the $561 \mathrm{~nm}$ laser power without changing the power of the $470 \mathrm{~nm}$ pulsed laser, leads increase of the green bleed through in the red channel that is completely eliminated by using FLCS in cells expressing free EGFP and mCherry (Fig.3-A, inset). In contrast, for cell expressing EGFP and mCherry in tandem, the residual cross-correlation amplitude after lifetime filters application does not change significantly (Figure 3B, inset). This result is important for the quantification of the amount of interacting proteins in living cells. Indeed, the quantity of spectral bleed through (and its repercussion to the cross-correlation amplitude) depends on both the relative concentration of green and red species (which is generally not controlled in live cells) and on the relative laser intensities used during the experiment. Concerning the latter, it is always more convenient to adapt green and red laser power during 
the experiment, for example to increase signal to noise ratio or to take care of the photobleaching. If we want to use a conventional methodology to get rid of spectral bleed through by subtracting its quantity to the measured cross-correlation amplitude (Bacia and Schwille, 2007), the two laser powers need to be defined before the experiment. In addition, the best would be to increase mCherry excitation in comparison to EGFP for minimizing the spectral bleed through but increasing the photobleaching of the mCherry for which the photostability is weak. Here, we show that the use of FLCS avoids all these difficulties and thus that this method is well adapted for the quantification of protein-protein interactions in living cells.

After bleed through correction, the apparent ratio of interacting EGFP and mCherry determined by FLCS in cells expressing the tandem molecule is only 0.30 ( $[\mathrm{gr}] /[\mathrm{g}]$ in Table 1). This low value is, however, expected due to the following aspects: (i) FRET occurs between the two fluorophores (EGFP and mCherry) in the tandem (Tramier et al., 2006) that concomitantly slightly decreases the amplitudes of red auto-correlation and the crosscorrelation resulting in the underestimation of the ratio (Hom et al., 2002); (ii) a known spectroscopic heterogeneity of mCherry is expected (Hilesheim et al., 2005; Padilla-Parra et al., 2009) resulting in the existence of a large fraction of dark protein (without matured chromophore) and thus of a large amount of apparent "free" EGFP. It was already shown by FLIM-FRET that the apparent maximum fraction of interacting donor (EGFP) in the mCherry-EGFP tandem is 0.45 (Padilla-Parra et al., 2009); (iii) the two color confocal volumes are slightly different relative to each other $\left(\omega_{0}\right.$ of $260 \mathrm{~nm}$ in the green channel and of $320 \mathrm{~nm}$ in the red channel, see Material and Methods), this will result in a diminution of the cross-correlation amplitude (Bacia and Schwille, 2007).

The auto- and cross-correlation curves obtained in cells expressing free EGFP and mCherry or the mCherry-EGFP tandem are fitted with a single species Brownian diffusion model for quantitative analysis and the results are summarized in Table 1. First, fit values confirm that using FLCS the amplitude of cross-correlation is canceled for freely diffusing EGFP and mCherry. This result is important for quantification since the spectral bleed through contribution $(0.25+/-0.12)$ is similar to the contribution of the cross-correlation amplitude for the mCherry-EGFP tandem $(0.30+/-0.15$ using FLCS $)$. Secondly, the diffusion coefficients of EGFP and mCherry determined by fit are in good agreement with other values reported in the literature (Brock et al., 1999; Ruan et al, 2002; Haustein et Schwille, 2003) regardless the different ways of calculating auto- and cross-correlation curves (with or without FLCS): i.e. $20 \mu \mathrm{m}^{2} \mathrm{~s}^{-1}$ for the monomeric fluorescent protein at $20^{\circ} \mathrm{C}$ in cells. Dual color FLCS does not introduce any distortion in auto-correlation curves and the fitted diffusion coefficients can be quantitatively interpreted. 


\section{CONCLUSION}

We show that dual color FLCS is capable of eliminating artifacts such as the cross-talk effect. Moreover, we stress the potentiality of the technique when applied to quantify protein-protein interactions in vivo. In this method, the cross-correlation amplitude does not depend on both the relative concentration of green and red species and on the relative laser intensities used during the experiment. In addition, the diffusion coefficients determined by fit are insensitive to lifetime filtering. The dual color FLCS experimental procedure where the different laser intensities do not have to be controlled during experiment is really very helpful to study quantitatively protein interactions in live sample. 


\section{Acknowledgement}

This work was performed at the Imaging facilities center of the Institut Jacques Monod and was supported by the Fondation pour la Recherche Médicale, the Région Ile de France (Soutien aux Equipes Scientifiques pour l'Acquisition de Moyens Expérimentaux), the Groupement Entreprises Françaises Lutte Contre Cancer, the Centre National de la Recherche Scientifique (Action Concertée Incitative Biologie Cellulaire, Moléculaire et Structurale), the Association pour la Recherche sur le Cancer, and the Association Nationale pour le Recherche. 


\section{REFERENCES}

Bacia K, Majoul IV, Schwille P. 2002. Probing the endocytic pathway in live cells using dual-color fluorescence cross-correlation analysis. Biophys J 83:1184-1193.

Bacia K, Schwille P. 2007. Practical guidelines for dual-color fluorescence crosscorrelation spectroscopy. Nat Protoc 2:2842-2856.

Berney C, Danuser G. 2003. FRET or no FRET: a quantitative comparison. Biophys J 84:3992-4010.

Böhmer M, Wahl M, Rahn H-J, Erdmann R, Enderlein J. 2002. Time-resolved fluorescence correlation spectroscopy. Chem Phys Lett 353:439-445.

Brock R, Vàmosi G, Vereb G, Jovin TM. 1999. Rapid characterization of green fluorescent protein fusion proteins on the molecular and cellular level by fluorescence correlation spectroscopy. Proc Natl Acad Sci USA 96:10123-10128.

Chen J, Irudayaraj J. 2010. Fluorescence Lifetime Cross Correlation Spectroscopy Resolves EGFR and Antagonist Interaction in Live Cells. Anal Chem 2010 Jun 29. [Epub ahead of print]

Elangovan M, Wallrabe H, Che Y, Day RN, Barroso M, Periasamy A. 2003. Characterization of one- and two-photon excitation fluorescence resonance energy transfer microscopy. Methods 29:58-73.

Förster T. 1948. Intermolecular energy migration and fluorescence. Annals phys. 6:55-75.

Gordon GW, Berry G, Liang XH, Levine B, Herman B. 1998. Quantitative fluorescence resonance energy transfer measurements using fluorescence microscopy. Biophys J 74: 27022713.

Gregor I, Enderlein J. 2007. Time-resolved methods in biophysics. 3. Fluorescence lifetime correlation spectroscopy. Photochem Photobiol Sci 6:13-18.

Haustien E, Schwille P. 2003. Ultrasensitive investigations of biological systems by fluorescence correlation spectroscopy. Methods 29:153-166.

Hilesheim LN, Y Chen, Müller JD. 2005. Dual-color photon counting histogram analysis of mRFP1 and EGFP in living cells. Biophys J 91:4273-4284.

Hom EFY, Verkman AS. 2002. Analysis of coupled bimolecular reaction kinetics and diffusion by two-color fluorescence correlation spectroscopy: enhanced resolution of kinetics by resonance energy transfer. Biophys J 83:533-546.

Huet S, Avilov SV, Ferbitz L, Daigle N, Cusack S, Ellenberg J. 2010. Nuclear import and assembly of influenza A virus RNA polymerase studied in live cells by fluorescence crosscorrelation spectroscopy. J Virol 84:1254-1264.

Kapusta P, Wahl M, Benda A, Hof M, Enderlein J. 2007. Fluorescence lifetime correlation spectroscopy. J Fluoresc 17:43-48.

Kogure T, Karasawa S, Araki T, Saito K, Kinjo M, Miyawaki A. 2006. A fluorescent variant of a protein from the stony coral Montipora facilitates dual-color single-laser fluorescence cross-correlation spectroscopy. Nat Biotech 24:577-581.

Lillemeier BF, Mörtelmaier MA, Forstner MB, Huppa JB, Groves JT, Davis MM. 2010. TCR and Lat are expressed on separate protein islands on T cell membranes and concatenate during activation. Nat Immunol 11:90-96.

Liu P, Sudhaharan T, Koh RML, Hwang LC, Ahmed S, Maruyama IN, Wohland T. 2007. Investigation of the dimerization of proteins from the epidermal growth factor receptor family by single wavelength fluorescence cross-correlation spectroscopy. Biophys J 93:684-698.

Maeder CI, Hink MA, Kinkhabwala A, Mayr R, Bastiaens PIH, Knop M. 2007. Spatial regulation of Fus3 MAP kinase activity through a reaction-diffusion mechanism in yeast pheromone signaling. Nat Cell Biol 9:1319-1326. 
Müller BK, Zaychikov E, Bräuchle C, Lamb DC. 2005. Pulsed interleaved excitation. Biophys J 89:3508-3522.

Padilla-Parra S, Audugé N, Coppey-Moisan M, Tramier M. 2008. Quantitative FRET analysis by fast acquisition time domain FLIM at high spatial resolution in living cells. Biophys J 95:2976-2988.

Padilla-Parra S, Audugé N, Lalucque H, Mevel JC, Coppey-Moisan M, Tramier M. 2009. Quantitative comparison of different fluorescent protein couples for fast FRET-FLIM acquisition. Biophys J 97:2368-2376.

Ray K, Zhang J, Lakowicz JR. 2008. Fluorescence lifetime correlation spectroscopic study of fluorophore-labeled silver nanoparticles. Anal Chem 80:7313-7318.

Ries J, Yu SR, Burkhardt M, Brand M, Schwille P. 2009. Modular scanning FCS quantifies receptor-ligand interactions in living multicellular organisms. Nat Meth 6:643-5.

Ruan Q, Chen Y, Gratton E, Glaser M, Mantulin WW. 2002. Cellular characterization of adenylate kinase and its isoform: two-photon excitation fluorescence imaging and fluorescence correlation spectroscopy. Biophys J 83:3177-3187.

Rüttinger S, Kapusta P, Patting M, Wahl M, Macdonald R. 2010. On the resolution capabilities and limits of fluorescence lifetime correlation spectroscopy (FLCS) measurements. J Fluoresc 20:105-114.

Savatier J, Jalaguier S, Ferguson ML, Cavaillès V, Royer CA. 2010. Estrogen receptor interactions and dynamics monitored in live cells by fluorescence cross-correlation spectroscopy. Biochem 49:772-781.

Stokholm D, Bartoli M, Sillon G, Bourg N, Davoust J, Richard I. 2004. Imaging calpain protease activity by multiphoton FRET in living mice. J Mol Biol 346:215-222.

Takahashi Y, Nishimura J, Suzuki A, Ishibashi K, Kinjo M, Miyawaki A. 2008. Crosstalk-free fluorescence cross-correlation spectroscopy by the switching method. Cell Struct Funct 33:143-150.

Thews E, Gerken M, Eckert R, Zäpfel J, Tietz C, Wrachtrup J. 2005. Cross talk free fluorescence cross correlation spectroscopy. Biophys J 89:2069-2076.

Tramier M, Gautier I, Piolot T, Ravalet S, Kemnitz K, Coppey J, Durieux C, Mignotte V, Coppey-Moisan M. 2002. Picosecond-hetero-FRET microscopy to probe protein-protein interactions in live cells. Biophys J 83:3570-3577.

Tramier M, Zahid M, Mevel JC, Masse MJ, Coppey-Moisan M. 2006. Sensitivity of CFP/YFP and GFP/mCherry pairs to donor photobleaching on FRET determination by fluorescence lifetime imaging microscopy in living cells. Microsc Res Tech 11:933-942.

Wouters FS, Bastiaens PI. 1999. Fluorescence lifetime imaging of receptor tyrosine kinase activity in cells. Curr Biol 9:1127-1130. 


\section{LEGEND TO FIGURES}

Figure 1: Dual color FLCS instrumentation.

A: Two lasers (470 $\mathrm{nm}$ pulsed and $561 \mathrm{~nm} \mathrm{CW}$ ) are conducted into epi-fluorescence microscope for live sample measurement. The confocal fluorescence selected by a pinhole is splited to green and red channels. Single photon counting detectors (SPADs) are used in timetagged time-resolved (TTTR) mode.

B: Fluorescence time-correlation histograms of GFP and mCherry fluorescence detected in the green (left) and red (right) channels by using $470 \mathrm{~nm}$ pulsed laser alone (upper), $561 \mathrm{~nm}$ CW laser (middle) and both (lower).

Figure 2: Fluorescence lifetime filters.

A: The experimental fluorescence time-correlated histogram (black) is a linear combination of the fluorescence decay (blue) and the background (pink). The residual curve corresponds to the error between experimental curve and linear combination.

B: Corresponding fluorescence lifetime filters of the fluorescence decay (blue) and the background calculated by Symphotime software (Picoquant, Berlin). These curves are respectively noted $f_{a}(i)$ and $f_{b}(i)$ in the text.

Figure 3: Dual color FLCS in live cell expressing EGFP and mCherry alone and in tandem. Experimental auto- and cross-correlation curves (dots) and fitting curves with a single species Brownian diffusion model (line) are presented for green auto-correlation (green), red autocorrelation (red), conventional cross-correlation (black) and lifetime filter cross-correlation (blue) for a representative cell expressing EGFP and mCherry in separate diffusion (A) and expressing EGFP and mCherry in co-diffusion (B). Inset in A and B are the conventional cross-correlation (solid line) and the lifetime filter cross-correlation (dotted line) for three consecutive measurements on the same focal volume by decreasing $561 \mathrm{~nm}$ laser power without changing the power of the $470 \mathrm{~nm}$ laser (from grey to black). 
Table 1: Diffusion time and cross-correlation ratio of EGFP-mCherry tandem and coexpressed separately

$$
\mathrm{D}_{\mathrm{g}}{ }^{\mathrm{a}} \quad \mathrm{D}_{\mathrm{r}}{ }^{\mathrm{a}} \quad \mathrm{D}_{\mathrm{gr}}{ }^{\mathrm{a}} \quad[\mathrm{gr}] /[\mathrm{g}]^{\mathrm{b}}
$$

$\begin{array}{cccccc}\begin{array}{c}\text { mCherry-EGFP } \\ \text { tandem }\end{array} & \text { without FLCS } & 11.6+/-2.9 & 10.7+/-3.4 & 11.9+/-3.7 & 0.56+/-0.10 \\ & \text { with FLCS } & 11.4+/-2.6 & 8.5+/-2.6 & 9.0+/-3.0 & 0.30+/-0.15 \\ \begin{array}{c}\text { Co-expressed } \\ \text { EGFP and mCherry }\end{array} & \text { without FLCS } & 20.4+/-2.8 & 24.9+/-5.1 & 17.5+/-5.2 & 0.25+/-0.12 \\ & \text { with FLCS } & 20.3+/-3.0 & 23.6+/-2.7 & - & 0\end{array}$

a, in $\mu \mathrm{m}^{2} . \mathrm{s}^{-1}$. b, calculated directly from $\mathrm{G}_{0 \mathrm{gr}} / \mathrm{G}_{0 \mathrm{rr}}$. Values $+/-$ sd from five independent experiments. 

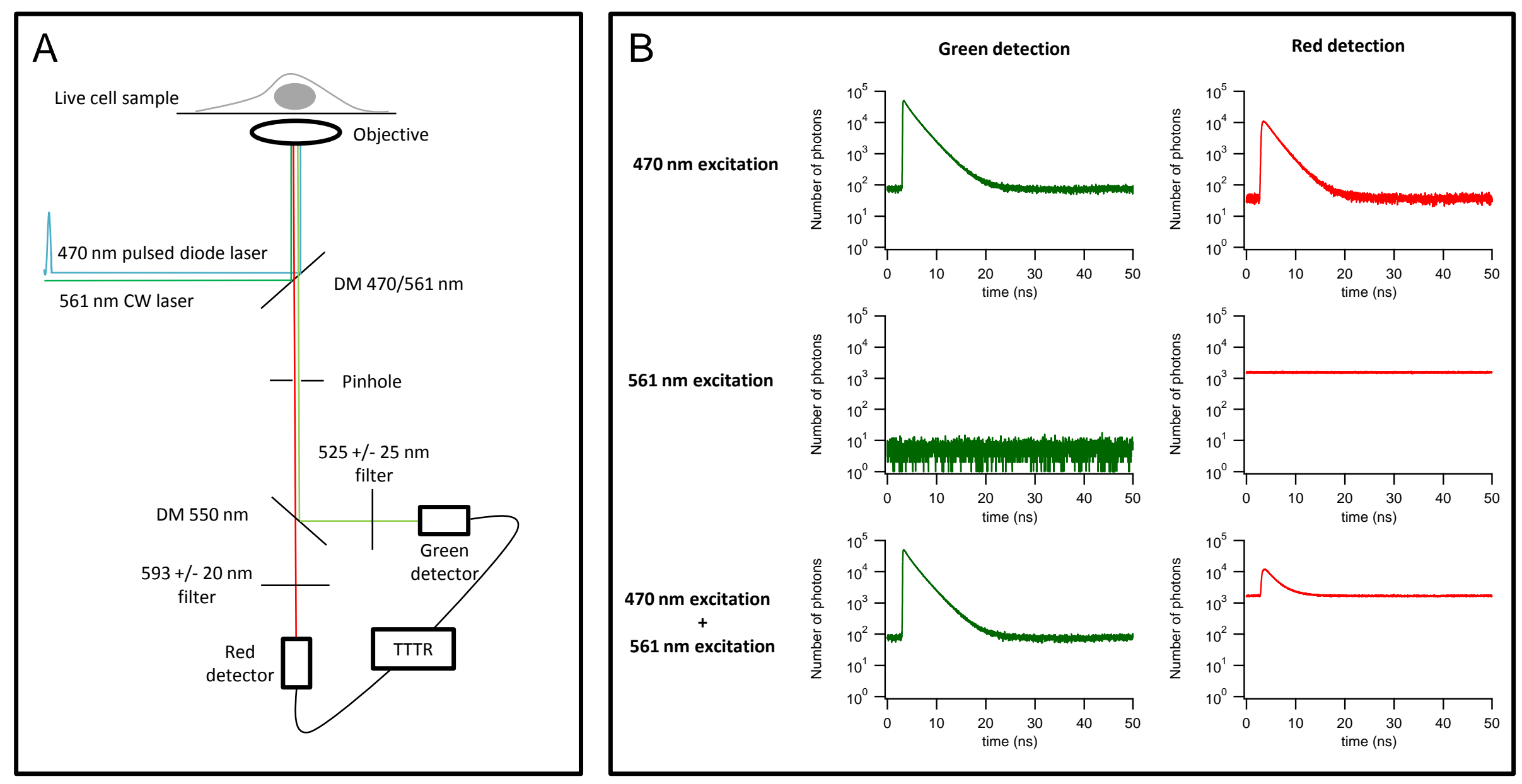

Padilla-Parra et al., Fig 1 


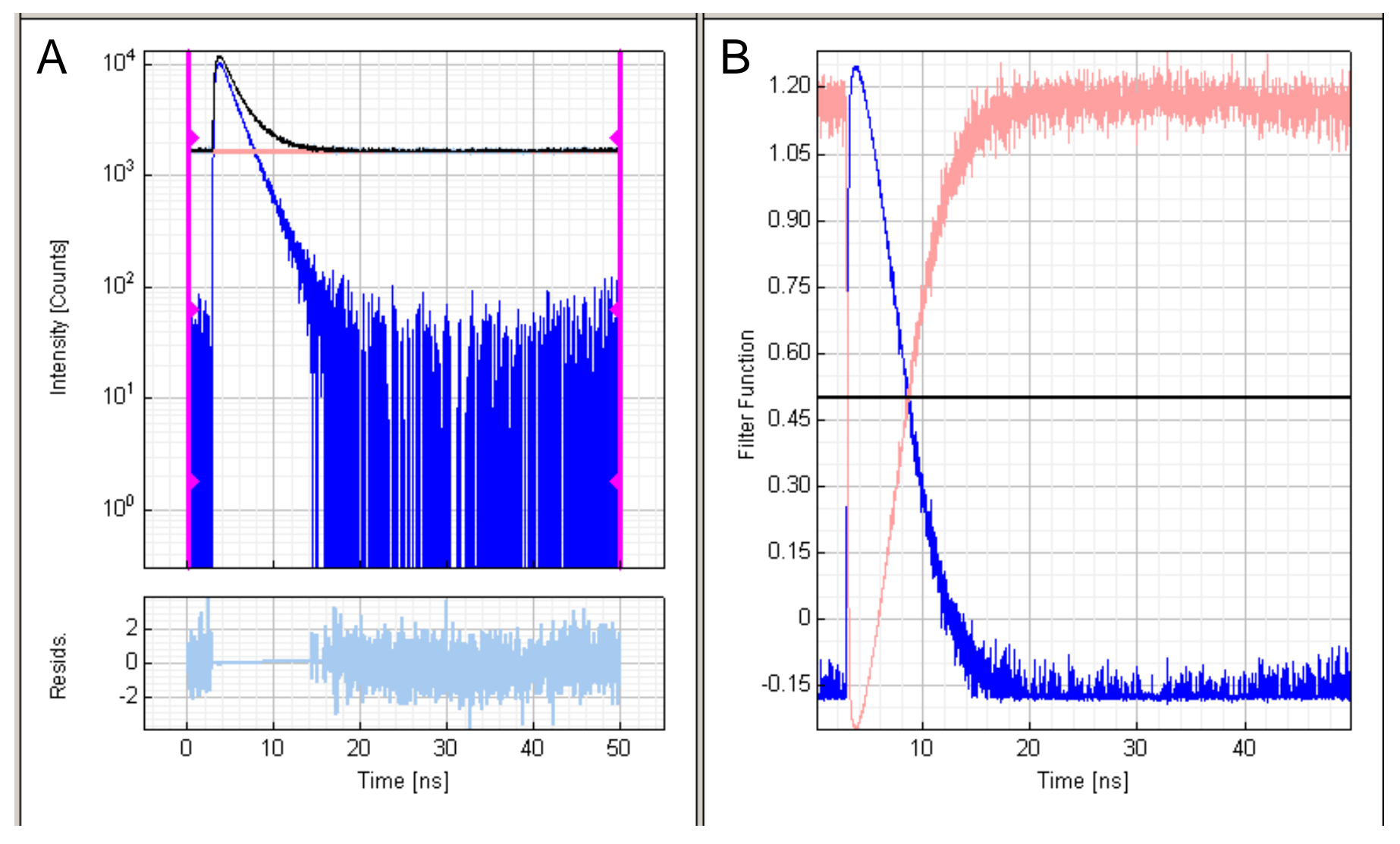

Padilla-Parra et al., Fig 2 


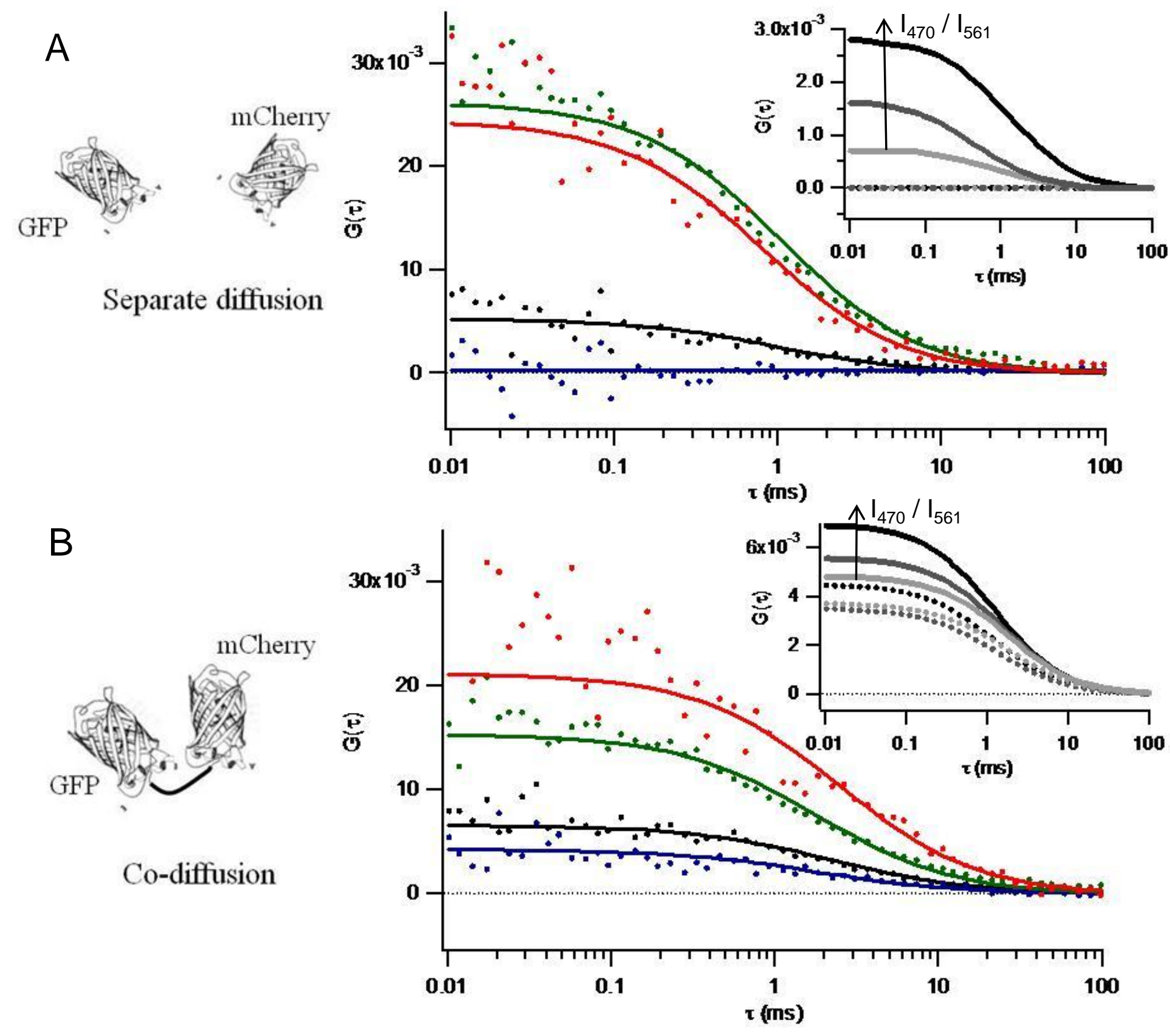

Padilla-Parra et al., Fig 3 